\title{
Fundamental impossibility theorems on voluntary participation in the provision of non-excludable public goods
}

\author{
Tatsuyoshi Saijo - Takehiko Yamato
}

Received: 9 October 2008 / Accepted: 11 November 2009 / Published online: 1 December 2009 (C) The Author(s) 2009. This article is published with open access at Springerlink.com

\begin{abstract}
Groves and Ledyard (Econometrica 45:783-809, 1977) constructed a mechanism attaining Pareto efficient allocations in the presence of public goods. After this path-breaking paper, many mechanisms have been proposed to attain desirable allocations with public goods. Thus, economists have thought that the free-rider problem is solved, in theory. Our view to this problem is not so optimistic. Rather, we propose fundamental impossibility theorems with public goods. In the previous mechanism design, it was implicitly assumed that every agent must participate in the mechanism that the designer provides. This approach neglects one of the basic features of public goods: non-excludability. We explicitly incorporate non-excludability and then show that it is impossible to construct a mechanism in which every agent has an incentive to participate.
\end{abstract}

We thank an anonymous referee and Takeshi Suzuki for useful comments. Research was partially supported by the Grant in Aid for Scientific Research of the Ministry of Education, Culture, Sports, Science and Technology in Japan. Yamato thanks the Division of the Humanities and Social Sciences at the California Institute of Technology for their hospitality during the period when this draft was written.

T. Saijo $(\bowtie)$

Institute of Social and Economic Research, Osaka University, Ibaraki, Osaka 567-0047, Japan

e-mail: saijo@iser.osaka-u.ac.jp

T. Saijo

CASSEL, UCLA, Los Angeles, CA 90095-1484, USA

T. Yamato

Department of Social Engineering, Graduate School of Decision Science and Technology,

Tokyo Institute of Technology, 2-12-1 Ookayama, Meguro-ku, Tokyo 152-8552, Japan

e-mail: yamato@valdes.titech.ac.jp 
Keywords Impossibility theorems - Voluntary participation · Non-excludable public goods · Lindahl equilibrium · Voluntary contribution mechanism · Olson's conjecture

\section{JEL Classification $\quad \mathrm{C} 72 \cdot \mathrm{D} 71 \cdot \mathrm{D} 78 \cdot \mathrm{H} 41$}

\section{Introduction}

Hurwicz (1972), in his path-breaking paper, showed that Walrasian mechanism has an incentive problem although many researchers at that time considered that it solves agents' incentive problem. That is, some agents have incentive not to reveal true excess demand functions. Later, Ledyard and Roberts (1974) showed the same problem in public good economies. In other words, it is impossible to design a mechanism that satisfies incentive compatibility where each agent reveals her true utility function or excess demand function as her dominant strategy in private or public good economies.

On the other hand, Groves and Ledyard (1977) designed a Nash implementable mechanism to achieve Pareto efficiency in the presence of public goods. Right after this discovery, Hurwicz (1979a) and Walker (1981) designed Nash implementable mechanisms for Lindahl allocations. Hereafter, many mechanisms having nice features have been proposed. Thus, economists have thought that the free-rider problem is solved, in theory.

Our view to this problem is not so optimistic. Rather, we propose fundamental impossibility theorems with public goods. In the previous mechanism design, it was implicitly assumed that every agent must participate in the mechanism that the designer provides. This approach neglects one of the basic features of public goods: non-excludability. In Saijo and Yamato (1999), we explicitly incorporated non-excludability in mechanism design by examining a two-stage game on voluntary participation in a mechanism for providing a non-excludable public good: in the first stage, each agent simultaneously decides whether or not to participate in the mechanism; and in the second stage, after knowing the other agents' participation decisions, the agents who chose participation in the first stage play the mechanism.

We fully characterized the equilibrium set of participants in the two-stage game for any second-stage mechanism satisfying symmetry, feasibility, and Pareto efficiency only for participants in symmetric Cobb-Douglas economies. ${ }^{1}$ In particular, we found that there exist economies for which full participation of all agents is not an equilibrium, implying that it is impossible to design reasonable mechanisms in which all agents always have participation incentives. The same negative result on voluntary participation holds for the voluntary contribution mechanism. In Saijo and Yamato (1999), however, we made a restrictive assumption that each agent has the same Cobb-Douglas utility function as well as the same endowment.

\footnotetext{
1 Our solution concept for the two-stage game assumes sequential rationality, although any equilibrium concept with complete information is allowed for the second stage. For example, if Nash equilibrium is the equilibrium concept for the second stage, then we examine subgame perfect Nash equilibria of the two-stage game.
} 
In this paper, we show the above negative results on participation incentives are robust in the sense that they occur in more general environments. We formulate full participation of all agents as an axiom on a mechanism called the voluntary participation condition: each agent always prefers participation to non-participation in the mechanism when all other agents participate in it.

First, we consider any mechanism implementing the Lindahl correspondence, called Lindhal mechanism. We show that any Lindahl mechanism fails to satisfy the voluntary participation condition in asymmetric Cobb-Douglas utility as well as quasi-linear utility economies in which agents may have different utility functions and endowments. Moreover, we identify the classes of Cobb-Douglas and quasi-linear utility economies for which the voluntary participation condition is satisfied. These classes become smaller and eventually vanish as the number of agents become larger, which can be interpreted as a support for Olson (1965) conjecture: a public good is less likely provided as the size of a group grows large.

The Lindahl correspondence satisfies individual rationality and Pareto efficiency, and moreover it is the only Nash implementable social choice correspondence that is Pareto efficient and individually rational under suitable conditions (Hurwicz 1979b). ${ }^{2}$ However, neither Pareto efficiency nor individual rationality is necessary to obtain a negative result on voluntary participation. We demonstrate that the voluntary contribution mechanism, which does not satisfy Pareto efficiency under Nash equilibrium, fails to meet the voluntary participation condition in asymmetric Cobb-Douglas and quasi-linear utility economies, even though the name of the mechanism contains the term "voluntary".

Moreover, we investigate a large class of mechanisms that are necessarily neither individually rational nor Pareto efficient. We establish impossibility results on voluntary participation in mechanisms meeting mild conditions. For the case of two agents, there is no feasible mechanism satisfying the voluntary participation condition and the Robinson Crusoe condition, which requires that if only one agent participates in the mechanism, then she choose an outcome that is best for her, on a domain of economies that include a Cobb-Douglas utility economy or a quasi-linear utility economy satisfying a certain condition. Furthermore, for the case of more than two agents, there is no feasible mechanism satisfying the voluntary participation condition and contribution monotonicity, which means that if $n-1$ agents chose participation and a new agent becomes a participant additionally, then the sum of contributions to the public good by the previous $n-1$ participants in the mechanism does not rise, on a sufficiently large domain of economies.

Palfrey and Rosenthal (1984), Moulin (1986), and Dixit and Olson (2000) studied the participation incentive problem in the provision of a public good. In those papers, however, the public good is discrete, while it is continuous in our model. Moreover, the mechanisms studied there are different from ours. Palfrey and Rosenthal (1984) examined voluntary contribution (or provision point) mechanisms with and without a refund to decide whether to produce a discrete public project or not. Contribu-

\footnotetext{
2 More specifically, Hurwicz (1979b) proved that if all Nash equilibrium allocations are Pareto efficient and individually rational, then every Lindahl allocation is a Nash allocation and every interior Nash allocation is a Lindahl allocation under appropriate assumptions on environments and mechanisms.
} 
tions are binary and making a fixed contribution can be interpreted as participation in a mechanism. They identified mixed strategy Nash equilibria of the mechanisms. Moulin (1986) used the no free ride axiom, requiring each agent have a participation incentive in a mechanism, to characterize the pivotal mechanism in economies with a discrete public good and quasi-linear preferences.

Dixit and Olson (2000) independently considered a two-stage participation game, similar to that in Saijo and Yamato (1999), from the viewpoint of the Coase theorem rather than mechanism design: in the first stage, each agent simultaneously decides whether to participate or not, and in the second stage, those who selected participation play a cooperative game of Coaseian bargaining with no costless enforcement of contracts. They examined a binary public good model like Palfrey and Rosenthal (1984). In particular, they found that the efficient equilibrium outcome of the participation game is not robust when introducing even very small transaction costs. This casts doubt on the validity of Coaseian claims of universal efficiency, which is similar to our negative view in the design of efficient resource allocation mechanisms with participation decisions.

The paper is organized as follows. In Sect. 2, we explain examples illustrating our basic idea. In Sect. 3, we introduce notation and definitions. We establish impossibility results on voluntary participation in Lindahl mechanisms in Sect. 4. In Sect. 5, we characterize a condition for which each agent loses a participation incentive in any Lindahl mechanism in a replica of a Cobb-Douglas or quasi-linear utility economy. In Sect. 6, we consider the participation problem on the voluntary contribution mechanism and a class of mechanisms satisfying mild conditions. In the final section, we make concluding remarks.

\section{Examples}

Let us consider the following two-agent economies with one private good $x$ and one pure public good $y$. Agent $i$ 's consumption bundle is denoted by $\left(x_{i}, y\right) \in \Re_{+}^{2}$ where $x_{i} \in \Re_{+}$is the level of private good she consumes on her own, and $y \in \Re_{+}$is the level of public good. Each agent has a Cobb-Douglas utility function: $u_{i}^{\alpha_{i}}\left(x_{i}, y\right)=$ $\alpha_{i} \ln x_{i}+\left(1-\alpha_{i}\right) \ln y$, where $\alpha_{i} \in(0,1)$ and $i=1,2$. Agent $i$ 's initial endowment is given by $\left(\omega_{i}, 0\right)$ for $i=1,2$, that is, there is no public good initially. However, the public good can be produced from the private good by means of a constant return to scale technology, and let $y=\sum_{i}\left(\omega_{i}-x_{i}\right)$ be the production function of the public good.

Consider any mechanism implementing the Lindahl correspondence (for example, see Hurwicz 1979a; Walker 1981; Hurwicz et al. 1984, and Tian 1990 for Nash implementation, ${ }^{3}$ and Moore and Repullo 1988 and Varian 1994 for subgame perfect implementation). Suppose that each agent is able to choose whether she participates in the mechanism. Then in order to achieve the desired Lindahl equilibrium allocation by using the mechanism, every agent must choose participation. Therefore, we ask a

\footnotetext{
3 The Lindahl correspondence is not Nash implementable due to the boundary problem, but the constrained Lindahl correspondence is Nash implementable.
} 
crucial question of whether each agent always has an incentive to participate in the mechanism. Unfortunately, our answer to this question is negative.

To see why, let $T \subseteq\{1,2\}$ be the set of agents who participate in the mechanism. An equilibrium allocation of the mechanism when the agents in $T$ participate in it is denoted by $\left(\left(x_{i}^{T}\right)_{i \in T}, y^{T}\right) .{ }^{4}$ If two agents decide to participate in the mechanism, then $\left(x_{1}^{\{1,2\}}, x_{2}^{\{1,2\}}, y^{\{1,2\}}\right)$ should be a Lindahl allocation of the economy consisting of two agents, since the mechanism implements the Lindahl correspondence. ${ }^{5}$ It is straightforward to check that there exists a unique Lindahl allocation given by $\left(x_{1}^{\{1,2\}}, x_{2}^{\{1,2\}}, y^{\{1,2\}}\right)=\left(\alpha_{1} \omega_{1}, \alpha_{2} \omega_{2}, \sum_{i=1,2}\left(1-\alpha_{i}\right) \omega_{i}\right)$.

Now suppose that some agent $i$ does not participate in the mechanism, while the other agent $j \neq i$ does, i.e., $T=\{j\}$. Then $\left(x_{j}^{\{j\}}, y^{\{j\}}\right)$ is a unique Lindahl allocation of the economy consisting of only one agent $j$. It is easy to see that $\left(x_{j}^{\{j\}}, y^{\{j\}}\right)=$ $\left(\alpha_{j} \omega_{j},\left(1-\alpha_{j}\right) \omega_{j}\right)$. Notice that non-participant $i$ can enjoy her initial endowment, $\omega_{i}$, as well as the non-excludable public good produced by agent $j \neq i, y^{\{j\}}$. On the other hand, she is no longer able to affect the decision on the provision of the public good. Because of this trade-off, it is not obvious whether or not each agent has an incentive to participate in the mechanism. The following condition should be satisfied if each agent has such a participation incentive:

$$
u_{i}^{\alpha_{i}}\left(x_{i}^{\{1,2\}}, y^{\{1,2\}}\right) \geq u_{i}^{\alpha_{i}}\left(\omega_{i}, y^{\{j\}}\right) \text { for } i, j=1,2, j \neq i,
$$

where $u_{i}^{\alpha_{i}}$ is any Cobb-Douglas utility function. We call condition (1) the voluntary participation condition. ${ }^{6}$

We show that no mechanism implementing the Lindahl correspondence satisfies this condition. This fact can be illustrated by using Kolm's triangle. See Fig. 1 in which $\left(\alpha_{1}, \alpha_{2}\right)=(0.5,0.7)$ and $\left(\omega_{1}, \omega_{2}\right)=(10,20)$. In this economy, agent 1 's valuation of the public good is higher than agent 2 's, but agent 1 is "poorer" than agent 2 . We will see that neither agent has a participation incentive. Point $A$ in Fig. 1 denotes the Lindahl equilibrium allocation when both agents participate in the mechanism: $A=\left(x_{1}^{\{1,2\}}, x_{2}^{\{1,2\}}, y^{\{1,2\}}\right)=(5,14,11)$. Point $B$ represents the allocation when agent 1 does not participate in the mechanism, but agent 2 does: $B=\left(\omega_{1}, x_{2}^{\{2\}}, y^{\{2\}}\right)=$ $(10,14,6)$. Since $u_{1}^{\alpha_{1}}\left(x_{1}^{\{1,2\}}, y^{\{1,2\}}\right) \approx 2.004<u_{1}^{\alpha_{1}}\left(\omega_{1}, y^{\{2\}}\right) \approx 2.047$ for $\alpha_{1}=0.5$, agent 1 prefers Point B to Point A and she does not participate in the

\footnotetext{
${ }^{4}$ Here we consider a general definition of a mechanism which specifies a strategy set of each participant in $T$ and an outcome function for each $T \subseteq\{1,2\}$.

5 A mechanism is said to implement the Lindahl correspondence if for each set of participants $T \subseteq\{1,2\}$ and each economy consisting of the participants in $T$, every equilibrium allocation is a Lindahl allocation and every Lindahl allocation is an equilibrium allocation.

6 The voluntary participation condition is different from the individually rational condition which requires that $u_{i}^{\alpha_{i}}\left(x_{i}^{\{1,2\}}, y^{\{1,2\}}\right) \geq u_{i}^{\alpha_{i}}\left(\omega_{i}, 0\right)$ for $i=1,2$. Since $u_{i}^{\alpha}\left(\omega_{i}, y^{\{j\}}\right) \geq u_{i}^{\alpha}\left(\omega_{i}, 0\right)$, the voluntary participation condition is stronger than the individually rational condition.
} 
Fig. 1 No Lindahl mechanism satisfies the voluntary participation condition when preferences are Cobb-Douglas

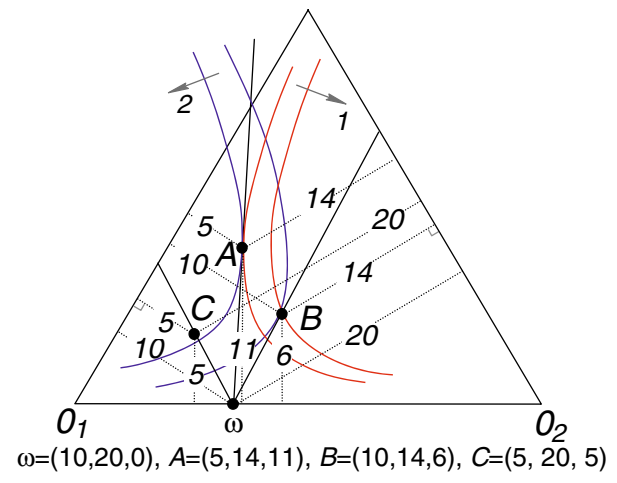

mechanism when agent 2 does. The same thing holds for agent 2. In Fig. 1, the allocation when agent 2 does not participate in the mechanism, but agent 1 does is represented by Point $C=\left(x_{1}^{\{1\}}, \omega_{2}, y^{\{1\}}\right)=(5,20,5)$. Agent 2 prefers Point $\mathrm{C}$ to Point A: $u_{2}^{\alpha_{2}}\left(x_{2}^{\{1,2\}}, y^{\{1,2\}}\right) \approx 2.567<u_{2}^{\alpha_{2}}\left(\omega_{2}, y^{\{1\}}\right) \approx 2.580$ for $\alpha_{2}=0.7$.

A similar negative result on voluntary participation in any Lindahl mechanism holds with quasi-linear preferences. Suppose that each agent has a quasi-linear utility function: $u_{i}^{\beta_{i}}\left(x_{i}, y\right)=x_{i}+\beta_{i} \ln y$, where $\beta_{i} \in\left(0, \omega_{i}\right)(i=1,2)$. It is easy to check that a unique Lindahl allocation when both agents participate in the mechanism is given by $\left(x_{1}^{\{1,2\}}, x_{2}^{\{1,2\}}, y^{\{1,2\}}\right)=\left(\omega_{1}-\beta_{1}, \omega_{2}-\beta_{2}, \sum_{i=1,2} \beta_{i}\right)$ and a unique Lindahl allocation when only one agent $j$ participates in it is $\left(x_{j}^{\{j\}}, y^{\{j\}}\right)=\left(\omega_{j}-\beta_{j}, \beta_{j}\right)$. The following voluntary participation condition should be satisfied if each agent has a participation incentive:

$$
u_{i}^{\beta_{i}}\left(x_{i}^{\{1,2\}}, y^{\{1,2\}}\right) \geq u_{i}^{\beta_{i}}\left(\omega_{i}, y^{\{j\}}\right) \text { for } i, j=1,2, j \neq i
$$

where $u_{i}^{\beta_{i}}$ is any quasi-linear utility function.

We will see that no Lindahl mechanism satisfies this condition. Suppose that $\left(\beta_{1}, \beta_{2}\right)=(2,3)$ and $\left(\omega_{1}, \omega_{2}\right)=(3,4)$. Then neither agent has a participation incentive. Point $A$ in Fig. 2 represents the Lindahl equilibrium allocation when both agents participate: $A=\left(x_{1}^{\{1,2\}}, x_{2}^{\{1,2\}}, y^{\{1,2\}}\right)=(1,1,5)$. Point $B$ stands for the allocation when agent 1 does not participate, but agent 2 does: $B=\left(\omega_{1}, x_{2}^{\{2\}}, y^{\{2\}}\right)=(3,1,3)$. Since $u_{1}^{\beta_{1}}\left(x_{1}^{\{1,2\}}, y^{\{1,2\}}\right) \approx 4.219<u_{1}^{\beta_{1}}\left(\omega_{1}, y^{\{2\}}\right) \approx 5.197$ for $\beta_{1}=2$, agent 1 prefers Point B to Point A, in other words, she has no participation incentive when agent 2 participates. The same thing holds for agent 2. In Fig. 2, the allocation when agent 2 does not participate, but agent 1 does is denoted by Point $C=$ $\left(x_{1}^{\{1\}}, \omega_{2}, y^{\{1\}}\right)=(1,4,2)$. Agent 2 prefers Point $\mathrm{C}$ to Point A: $u_{2}^{\beta_{2}}\left(x_{2}^{\{1,2\}}, y^{\{1,2\}}\right) \approx$ $5.828<u_{2}^{\beta_{2}}\left(\omega_{2}, y^{\{1\}}\right) \approx 6.079$ for $\beta_{2}=3$. 
Fig. 2 No Lindahl mechanism satisfies the voluntary participation condition when preferences are quasi-linear

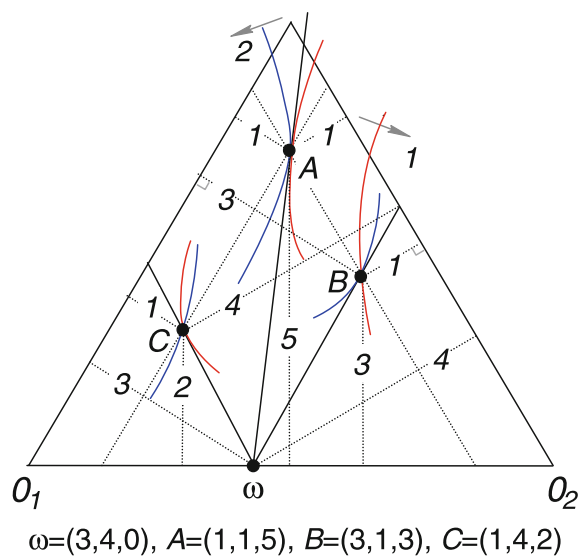

\section{Notation and definitions}

In the previous section, we see that any Lindahl mechanism fails to satisfy the voluntary participation condition in economies with two agents by looking at certain values of Cobb-Douglas and quasi-linear preference and endowment parameters. We will show similar negative results hold for any number of agents. Also, we will identify classes of preference and endowment parameters for which agents lose participation incentives. In particular, these classes become larger as the number of agents increases.

First of all, we introduce notation and definitions. As in Sect. 2, there are one private good $x$ and one public good $y$ with a constant return to scale technology. Let $N=\{1,2, \ldots, n\}$ be the set of agents, with generic element $i$. Each agent $i$ 's preference relation admits a numerical representation $u_{i}: \Re_{+}^{2} \rightarrow \Re$ which is continuously differentiable, strictly quasi-concave, and strictly monotonic. Let $U_{i}$ be the class of utility functions admissible for agent $i$ and $U \equiv \prod_{i \in N} U_{i}$. Agent $i$ 's initial endowment is denoted by $\left(\omega_{i}, 0\right)$. There is no public good initially. Let $\Omega_{i}$ be the class of private good endowments admissible for agent $i$ and $\Omega \equiv \prod_{i \in N} \Omega_{i}$. An economy is a list of utility functions and endowments of all agents, $e=(u, \omega)=\left(\left(u_{i}\right)_{i \in N},\left(\omega_{i}\right)_{i \in N}\right)$ and the class of admissible economies is denoted by $E=U \times \Omega$.

Let an economy $e=(u, \omega) \in E$ be given. Also, let $P(N)$ be the collection of all no-empty subsets of $N$. Given $T \in P(N), e_{T}=\left(u_{T}, \omega_{T}\right)=\left(\left(u_{i}\right)_{i \in T},\left(\omega_{i}\right)_{i \in T}\right)$ is a sub-economy consisting of agents in $T$. A feasible allocation for $e_{T}$ is a list $\left(x_{T}, y\right) \equiv$ $\left(\left(x_{i}\right)_{i \in T}, y\right) \in \mathfrak{R}_{+}^{\# T+1}$ such that $\sum_{i \in T}\left(\omega_{i}-x_{i}\right)=y$. The set of feasible allocations for $e_{T}$ is denoted by $A\left(e_{T}\right)$.

A mechanism is a function $\Gamma$ that associates with each $T \in P(N)$ a pair $\Gamma(T)=$ $\left(S^{T}, g^{T}\right)$, where $S^{T}=\times_{i \in T} S_{i}^{T}$ and $g^{T}: S^{T} \rightarrow \Re^{\# T+1}$. Here $S_{i}^{T}$ is the strategy space of agent $i \in T$ and $g^{T}$ is the outcome function when the agents in $T$ play the mechanism. Given $g^{T}(s)=\left(x_{T}, y\right)$, let $g_{i}^{T}(s) \equiv\left(x_{i}, y\right)$ for $i \in T$ and $g_{y}^{T}(s)=y$. The allocation for the entire economy when the agents in $T \in P(N)$ participate in the mechanism is provided by $\left(x_{T},\left(\omega_{i}\right)_{i \in N-T}, y\right)$, that is, every non-participant enjoys her endowment $\omega_{i}$ and the public good $y$ produced by the participants. Notice that 
we assume neither individual feasibility $\left(g^{T}(s) \in \mathfrak{R}_{+}^{\# T+1}\right.$ for all $\left.s \in S^{T}\right)$ nor balancedness $\left(g^{T}(s) \in A\left(e_{T}\right)\right.$ for all $\left.s \in S^{T}\right)$. Our negative results hold without requiring these conditions.

An equilibrium correspondence is a correspondence $\mu$ which associates with each mechanism $\Gamma$, each economy $e \in E$, and each set of agents $T \in P(N)$, a set of strategy profiles $\mu_{\Gamma}\left(e_{T}\right) \subseteq S^{T}$, where $\left(S^{T}, g^{T}\right)=\Gamma(T)$. The set of $\mu$-equilibrium allocations of $\Gamma$ for $e_{T}$ is denoted by $g^{T} \circ \mu_{\Gamma}\left(e_{T}\right) \equiv\left\{\left(x_{T}, y\right) \in \mathfrak{R}^{\# T+1} \mid\right.$ there exists $s \in S^{T}$ such that $s \in \mu_{\Gamma}\left(e_{T}\right)$ and $\left.g^{T}(s)=\left(x_{T}, y\right)\right\}$, where $\left(S^{T}, g^{T}\right)=\Gamma(T)$. In this paper, we consider an arbitrary equilibrium correspondence with complete information among agents. Examples of equilibrium correspondences include the Nash equilibrium correspondence, the strong Nash equilibrium correspondence, and any refinement of the Nash equilibrium correspondence such as the perfect and proper equilibrium correspondences.

Given an economy $e=(u, \omega) \in E$ and a set of agents $T \in P(N)$, a feasible allocation $\left(x_{T}, y\right) \in A\left(e_{T}\right)$ is a Lindahl allocation for $e_{T}$ if there is a price vector $p \in \mathfrak{R}_{+}^{\# T}$ such that for each agent $i \in T, x_{i}+p_{i} y=\omega_{i}$ and $u_{i}\left(x_{i}, y\right) \geq u_{i}\left(x_{i}^{\prime}, y^{\prime}\right)$ for any $\left(x_{i}^{\prime}, y^{\prime}\right) \in \mathfrak{R}_{+}^{2}$ such that $x_{i}^{\prime}+p_{i} y^{\prime} \leq \omega_{i}$. Let $L\left(e_{T}\right)$ be the set of Lindahl allocations for $e_{T}$.

Let an equilibrium correspondence $\mu$ be given. A Lindahl mechanism under $\mu$ is a mechanism such that for each economy $e=(u, \omega) \in E$ and each set of agents $T \in P(N), g^{T} \circ \mu_{\Gamma}\left(e_{T}\right)=L\left(e_{T}\right)$.

A Lindahl mechanism under $\mu$ is a mechanism implementing the Lindahl correspondence in $\mu$-equilibrium, that is, for each set of participants $T \in P(N)$ and each economy consisting of the participants in $T$, every $\mu$-equilibrium allocation is a Lindahl allocation and every Lindahl allocation is a $\mu$-equilibrium allocation. The above definition of a mechanism implementing the Lindahl correspondence is a generalization of the usual one, in which all agents are supposed to participate, to the case in which voluntary participation is allowed.

\section{Impossibility results on voluntary participation in Lindahl mechanisms}

We introduce the following condition on voluntary participation in mechanisms. Let an equilibrium correspondence $\mu$ be given.

Definition 1 The mechanism $\Gamma$ satisfies voluntary participation for an economy $e=(u, \omega)$ under $\mu$ if for all $\left(x^{N}, y^{N}\right) \in g^{N} \circ \mu_{\Gamma}\left(e_{N}\right)$ and all $i \in N$,

$$
u_{i}\left(x_{i}^{N}, y^{N}\right) \geq u_{i}\left(\omega_{i}, y_{\min }^{N-\{i\}}\right)
$$

where $y_{\min }^{N-\{i\}} \in \underset{y^{N-\{i\}} \in g_{y}^{N-\{i\}} \circ \mu_{\Gamma}\left(e_{N-\{i\}}\right)}{\arg \min } u_{i}\left(\omega_{i}, y^{N-\{i\}}\right)$. Also, the mechanism $\Gamma$ satisfies voluntary participation on the class of economies $E$ under $\mu$ if it satisfies voluntary participation for all economies $e=(u, \omega) \in E$ under $\mu$. 
Since there is one public good and preferences satisfy monotonicity, $y_{\min }^{N-\{i\}}$ is the minimum equilibrium level of public good when all agents except $i$ participate in the mechanism. Consider an agent who decides not to participate in the mechanism. Then she can enjoy the non-excludable public good produced by the other agents without providing any private good, while she cannot affect the decision on the provision of the public good. Voluntary participation requires that no agent can benefit from such a free-riding action. Note that when an agent chooses non-participation, she has a pessimistic view on the outcome of her action: an equilibrium outcome that is most unfavorable for her will occur. Moulin (1986) proposed a similar condition, called the No Free Ride axiom, when public goods are discrete and costless, and preferences are quasi-linear.

We will show any Lindahl mechanism fails to satisfy the voluntary participation condition under mild conditions. First of all, consider the class of Cobb-Douglas utility economies: $E^{C D} \equiv\left\{\left(\left(u_{i}\right)_{i \in N},\left(\omega_{i}\right)_{i \in N}\right) \mid \forall i \in N, u_{i}\left(x_{i}, y\right)=u_{i}^{\alpha_{i}}\left(x_{i}, y\right)=\right.$ $\left.\alpha_{i} \ln x_{i}+\left(1-\alpha_{i}\right) \ln y, \alpha_{i} \in(0,1), \omega_{i} \in \Re_{++}\right\}$. Such an economy is specified by a list of Cobb-Douglas preference parementers and endowments of $n$ agents, $(\alpha, \omega) \equiv$ $\left(\left(\alpha_{1}, \ldots, \alpha_{n}\right),\left(\omega_{1}, \ldots, \omega_{n}\right)\right)$ such that $\alpha_{i} \in(0,1)$ and $\omega_{i} \in(0, \bar{\omega}]$ for all $i$. Here $\bar{\omega}$ is the upper bound of each endowment. Without loss of generality, we assume that $\bar{\omega}=1$. Hence, the set of economies is represented by the product of intervals $E^{C D}=(0,1)^{n} \times(0,1]^{n}$ endowed with Lebesgue measure $\lambda$.

Let $\alpha(n) \in(0,1)$ be a unique value satisfying $\alpha \ln \alpha /(\alpha-1)=\ln (n /(n-1))$. We have the following negative result on voluntary participation regarding Cobb-Douglas utility economies.

Theorem 1 Let $(\alpha, \omega) \in E^{C D}$ be any Cobb-Douglas utility economy in which $\alpha_{i}>$ $\alpha(n)$ for agent $i$ such that $\left(1-\alpha_{i}\right) \omega_{i} \leq\left(1-\alpha_{j}\right) \omega_{j}$ for all $j \neq i$. Then any Lindahl mechanism fails to satisfy voluntary participation for $(\alpha, \omega)$ under $\mu$.

Proof Fix any $(\alpha, \omega) \in E^{C D}$. For each $T \in P(N)$, it is easy to check there exists a unique Lindahl equilibrium allocation for $\left(\alpha_{i}, \omega_{i}\right)_{i \in T}$, which coincides with a unique $\mu$-equilibrium allocation of the mechanism when agents in $T$ participate in it, given by $\left(x_{i}^{T}, y^{T}\right)=\left(\alpha_{i} \omega_{i}, \sum_{j \in T}\left(1-\alpha_{j}\right) \omega_{j}\right)$. Therefore, the difference between agent $i$ 's utility level when all agents participate in the mechanism and that when all agents except $i$ participate in it is given by

$$
\begin{aligned}
\Delta u_{i}(\alpha, \omega) \equiv u_{i}^{\alpha_{i}}\left(x_{i}^{N}, y^{N}\right)-u_{i}^{\alpha_{i}}\left(\omega_{i}, y^{N-\{i\}}\right) & \left\{\ln \left[\left(1-\alpha_{i}\right) \omega_{i}+\sum_{j \neq i}\left(1-\alpha_{j}\right) \omega_{j}\right]\right. \\
=\alpha_{i} \ln \alpha_{i}+\left(1-\alpha_{i}\right) & \left.-\ln \left[\sum_{j \neq i}\left(1-\alpha_{j}\right) \omega_{j}\right]\right\} .
\end{aligned}
$$

Take $i \in N$ such that $\left(1-\alpha_{i}\right) \omega_{i} \leq\left(1-\alpha_{j}\right) \omega_{j}$ for all $j \neq i$. Without loss of generality, let $i=1$. We prove that $\Delta u_{1}(\alpha, \omega)<0$ if $\alpha_{1}>\alpha(n)$, so that the voluntary 
participation condition is violated. Since $(n-1)\left(1-\alpha_{1}\right) \omega_{1} \leq \sum_{j \neq 1}\left(1-\alpha_{j}\right) \omega_{j}$,

$$
\begin{gathered}
\Delta u_{1}(\alpha, \omega) \leq \alpha_{1} \ln \alpha_{1}+\left(1-\alpha_{1}\right)\left\{\ln \left[(1 /(n-1)+1) \sum_{j \neq 1}\left(1-\alpha_{j}\right) \omega_{j}\right]\right. \\
\left.-\ln \left[\sum_{j \neq 1}\left(1-\alpha_{j}\right) \omega_{j}\right]\right\} \\
=\left(1-\alpha_{1}\right)\left[\ln (n /(n-1))-\alpha_{1} \ln \alpha_{1} /\left(\alpha_{1}-1\right)\right] .
\end{gathered}
$$

Let $h(\alpha) \equiv \alpha \ln \alpha /(\alpha-1)$. First, we show that $h(\alpha)$ is strictly increasing in $\alpha \in$ $(0,1)$. Note that $d h(\alpha) / d \alpha=(\alpha-1-\ln \alpha) /(\alpha-1)^{2}$. Since $(\alpha-1)^{2}>0$, it remains to prove that $B(\alpha) \equiv \alpha-1-\ln \alpha>0$. It is easy to check that $d B(\alpha) / d \alpha<0$ if $\alpha \in(0,1), d B(\alpha) / d \alpha=0$ if $\alpha=1$, and $B(1)=0$. Therefore, $B(\alpha)>0$ for $\alpha \in(0,1)$.

Second, by $L^{\prime}$ Hôpital's rule, $\lim _{\alpha \rightarrow 0} h(\alpha)=\lim _{\alpha \rightarrow 0}[\ln \alpha /\{1-1 / \alpha\}]=$ $\lim _{\alpha \rightarrow 0}[\alpha]=0$ and $\lim _{\alpha \rightarrow 1} h(\alpha)=\lim _{\alpha \rightarrow 1}[\alpha]=1$. Since $d h(\alpha) / d \alpha>0$ and $\ln (n /(n-1))<1$ for $n \geq 2$, it follows that there exists a unique $\alpha(n) \in(0,1)$ such that $h(\alpha(n))=\ln (n /(n-1))$ and $h(\alpha)>\ln (n /(n-1))$ for $\alpha>\alpha(n)$, implying that $\Delta u_{1}(\alpha, \omega)<0$ if $\alpha_{1}>\alpha(n)$.

Each agent contributes $\left(1-\alpha_{i}\right) \omega_{i}$ of the private good to the production of the public good in a Lindahl allocation for a Cobb-Doulas utility economy (see the proof of Theorem 1). Consider agent $i$ such that $\left(1-\alpha_{i}\right) \omega_{i} \leq\left(1-\alpha_{j}\right) \omega_{j}$ for all $j \neq i$, that is, agent $i$ 's contribution to the public good is the minimum and hence the reduction in the public good provision level by agent $i$ 's non-participation is the lowest among agents. Theorem 1 says that if this agent's value of the private good relative to the public good is large enough $\left(\alpha_{i}>\alpha(n)\right)$, then she has a non-participation incentive and the voluntary participation condition is violated. By Theorem 1, if the class of admissible economies $E$ contains a Cobb-Douglas utility economy satisfying the above condition, then any Lindahl mechanism fails to satisfy voluntary participation on $E$.

Table 1 illustrates how the value of $\alpha(n)$ depends on the number of agents, $n$. Since $\alpha(n)$ is strictly decreasing in $n$ and $\lim _{n \rightarrow \infty} \alpha(n)=0$, the measure of the set of CobbDouglas utility economies for which any Lindahl mechanism satisfies the voluntary participation condition becomes smaller and converges to zero, as the number of agents grows large. ${ }^{7}$ As Olson (1965) asserted, a public good would be less likely provided as the size of an economy becomes larger. The above result verifies this conjecture from the perspective of a participation incentive for any Lindahl mechanism in asymmetric Cobb-Douglas economies.

A similar negative result holds for quasi-linear utility economies. Let $E^{Q L} \equiv$ $\left\{\left(\left(u_{i}\right)_{i \in N},\left(\omega_{i}\right)_{i \in N}\right) \mid \forall i \in N, u_{i}\left(x_{i}, y\right)=u_{i}^{\beta_{i}}\left(x_{i}, y\right)=x_{i}+\beta_{i} \ln y, \beta_{i} \in\left(0, \omega_{i}\right)\right\}$ be

\footnotetext{
7 The condition in Theorem 1 for which the voluntary participation condition is violated becomes identical to that discussed in Saijo and Yamato (1999) if each agent has the same Cobb-Douglas utility function and endowment.
} 
Table 1 The value of $\alpha(n)$

\begin{tabular}{llllllllll}
\hline$n$ & 2 & 3 & 4 & 5 & 10 & 50 & 100 & 500 & 1000 \\
\hline$\alpha(n)$ & 0.50000 & 0.20246 & 0.11910 & 0.08186 & 0.02886 & 0.00357 & 0.00155 & 0.00024 & 0.00011 \\
\hline
\end{tabular}

the class of quasi-linear utility economies. Here we assume that $\omega_{i}>\beta_{i}$ for each $i$ to ensure an interior solution. Such an economy is specified by a list of quasi-linear preference parementers and endowments of $n$ agents, $(\beta, \omega) \equiv\left(\left(\beta_{1}, \ldots, \beta_{n}\right),\left(\omega_{1}, \ldots, \omega_{n}\right)\right)$ such that $\beta_{i} \in\left(0, \omega_{i}\right)$ and $\omega_{i} \in(0,1]$ for all $i$. In this case, the set of economies is given by $E^{Q L} \equiv\left\{(\beta, \omega) \in(0,1)^{n} \times(0,1]^{n}: \beta_{i}<\omega_{i}, \forall i\right\}$.

Theorem 2 Let $(\beta, \omega) \in E^{Q L}$ be an arbitrary quasi-linear utility economy. Any Lindahl mechanism fails to satisfy voluntary participation for $(\beta, \omega)$ under $\mu$.

Proof Fix any $(\beta, \omega) \in E^{Q L}$. For each $T \in P(N)$, it is easy to check there exists a unique Lindahl equilibrium allocation at $\left(\beta_{i}, \omega_{i}\right)_{i \in T}$, which coincides with a unique $\mu$-equilibrium allocation of the mechanism when agents in $T$ participate in it, given by $\left(x_{i}^{T}, y^{T}\right)=\left(\omega_{i}-\beta_{i}, \sum_{j \in T} \beta_{j}\right)$. Therefore, the difference between agent $i$ 's utility level when all agents participate in the mechanism and that when all agents except $i$ participate in it is given by

$$
\begin{aligned}
\Delta u_{i}(\beta) & \equiv u_{i}^{\beta_{i}}\left(x_{i}^{N}, y^{N}\right)-u_{i}^{\beta_{i}}\left(\omega_{i}, y^{N-\{i\}}\right) \\
& =\beta_{i}\left\{-1+\ln \left[\beta_{i}+\sum_{j \neq i} \beta_{j}\right]-\ln \left[\sum_{j \neq i} \beta_{j}\right]\right\} .
\end{aligned}
$$

We will show that there exist some $i$ such that $\Delta u_{i}(\beta)<0$. Take $i \in N$ such that $\beta_{i} \leq \beta_{j}$ for all $j \neq i$. Without loss of generality, let $i=1$. Since $(n-1) \beta_{1} \leq \sum_{j \neq 1} \beta_{j}$,

$$
\begin{aligned}
\Delta u_{1}(\beta) & \leq \beta_{1}\left\{-1+\ln \left[(1 /(n-1)+1) \sum_{j \neq 1} \beta_{j}\right]-\ln \left[\sum_{j \neq 1} \beta_{j}\right]\right\} \\
& =\beta_{1}\{-1+\ln n-\ln (n-1)\} .
\end{aligned}
$$

Since the function $\ln n-\ln (n-1)$ is decreasing in $n$ and $\ln 2-\ln 1 \approx 0.693<1$, it follows from the above inequality that $\Delta u_{1}(\beta)<0$ for $n \geq 2$. Therefore, the voluntary participation condition is violated.

By Theorem 2, if the class of admissible economies $E$ contains a quasi-linear utility economy $(\beta, \omega) \in E^{Q L}$, then any Lindahl mechanism fails to satisfy voluntary participation on $E$. Notice that Theorem 2 holds without making any condition on parameters of quasi-linear utility functions, on endowments, nor on the number of agents, except that the endowment of each agent is large enough to guarantee an interior solution (i.e., $\omega_{i}>\beta_{i}$ ). In this sense, the result for quasi-linear utility economies is stronger than Theorem 1 for Cobb-Douglas utility economies. 


\section{A participation incentive of each agent in a replica economy}

In the previous section, we show that there exist Cobb-Douglas and quasi-linear utility economies for which some agent fails to have a participation incentive in any Lindahl mechanism, so that the mechanism does not satisfy the voluntary participation condition. In this section, we check whether or not each agent has a participation incentive in any Lindahl mechanism in a replica of any given Cobb-Douglas or quasi-linear utility economy. We will find that in a sufficiently large replica of any economy of $n$-type agents, every type of agent has no participation incentive.

Let $\mu$ be any equilibrium correspondence, $\Gamma$ be any Lindahl mechanism under $\mu$, and $(\alpha, \omega) \in E^{C D}$ be any Cobb-Douglas utility economy of $n$-type agents. Consider the $k$-replica of this economy in which there are $k$ agents of type $\left(\alpha_{i}, \omega_{i}\right)$ for each $i \in N$. Denote the set of all $k n$ agents in the $k$-replica economy by $k N$. Let $\left(x_{i}^{k N}, y^{k N}\right)$ be the consumption bundle each agent of type $\left(\alpha_{i}, \omega_{i}\right)$ receives at the unique Lindahl allocation for $k N$ and $y^{k N-\{i\}}$ be the public good level at the unique Lindahl allocation for $k N-\{i\}$. Also, let

$$
\Delta u_{i}(\alpha, \omega, k) \equiv u_{i}^{\alpha_{i}}\left(x_{i}^{k N}, y^{k N}\right)-u_{i}^{\alpha_{i}}\left(\omega_{i}, y^{k N-\{i\}}\right)
$$

be the difference between the utility level of each agent of type $\left(\alpha_{i}, \omega_{i}\right)$ when all agents participate in the mechanism $\Gamma$ and that when all agents except her, that is, $k-1$ agents of type $\left(\alpha_{i}, \omega_{i}\right)$ as well as $k(n-1)$ agents of other types participate in $\Gamma$ in the $k$-replica economy. If the mechanism $\Gamma$ satisfies the voluntary participation condition for the $k$-replica economy, then for any $(\alpha, \omega) \in E^{C D}$ and for any $i \in N$, we must have $\Delta u_{i}(\alpha, \omega, k) \geq 0$. However, we have the following negative result. Given any $n \geq 2$, any $(\alpha, \omega) \in E^{C D}$, and any $i \in N$, let $k_{i}(\alpha, \omega)$ be the largest integer less than or equal to $e^{h\left(\alpha_{i}\right)}\left(1-\alpha_{i}\right) \omega_{i} /\left\{\left(e^{h\left(\alpha_{i}\right)}-1\right) \sum_{j=1}^{n}\left(1-\alpha_{j}\right) \omega_{j}\right\}$, where $e$ is the base of the natural logarithm and $h\left(\alpha_{i}\right) \equiv \alpha_{i} \ln \alpha_{i} /\left(\alpha_{i}-1\right)$.

Theorem 3 Consider an arbitrary Lindahl mechanism $\Gamma$ under $\mu$. Given any $n \geq 2$, any $(\alpha, \omega) \in E^{C D}$, and any $i \in N, \Delta u_{i}(\alpha, \omega, k) \geq 0$ for any positive integer $k \leq k_{i}(\alpha, \omega)$; and $\Delta u_{i}(\alpha, \omega, k)<0$ for any positive integer $k>k_{i}(\alpha, \omega)$.

Proof It is not hard to check that $\left(x_{i}^{k N}, y^{k N}\right)=\left(\alpha_{i} \omega_{i}, k \sum_{j \in N}\left(1-\alpha_{j}\right) \omega_{j}\right)$ and $y^{k N-\{i\}}=(k-1)\left(1-\alpha_{i}\right) \omega_{i}+k \sum_{j \neq i}\left(1-\alpha_{j}\right) \omega_{j}$. Therefore,

$$
\begin{aligned}
\Delta u_{i}(\alpha, \omega, k)= & \alpha_{i} \ln \alpha_{i} \\
& +\left(1-\alpha_{i}\right)\left\{\ln \left[k\left(1-\alpha_{i}\right) \omega_{i}+k \sum_{j \neq i}\left(1-\alpha_{j}\right) \omega_{j}\right]\right. \\
& \left.-\ln \left[(k-1)\left(1-\alpha_{i}\right) \omega_{i}+k \sum_{j \neq i}\left(1-\alpha_{j}\right) \omega_{j}\right]\right\} .
\end{aligned}
$$




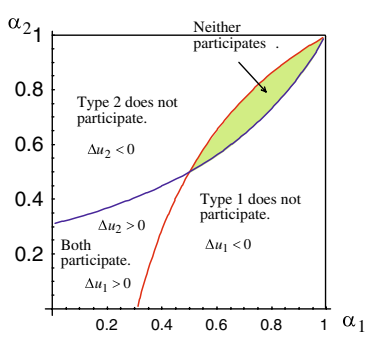

(a) The $k=1$-replica.

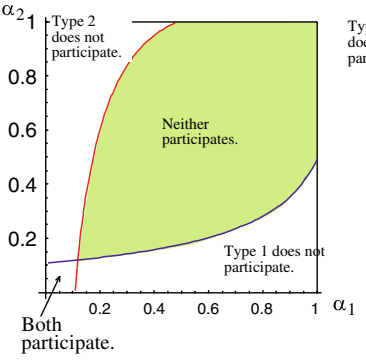

(b) The $k=2$-replica.

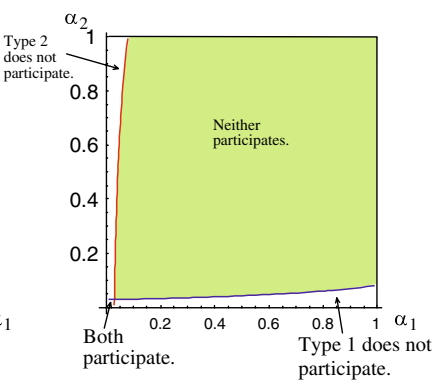

(c) The $k=5$-replica.

Fig. 3 Participation incentives in two-agent Cobb-Dogulas utiltiy economies with symmetric endowments

Let $k_{i}^{*}(\alpha, \omega)$ be a value satisfying the equation $\Delta u_{i}\left(\alpha, \omega, k_{i}^{*}(\alpha, \omega)\right)=0$. This equation can rewritten as

$$
\begin{aligned}
& \ln \left[\frac{k_{i}^{*}(\alpha, \omega)\left(1-\alpha_{i}\right) \omega_{i}+k_{i}^{*}(\alpha, \omega) \sum_{j \neq i}\left(1-\alpha_{j}\right) \omega_{j}}{\left.\left(k_{i}^{*}(\alpha, \omega)-1\right)\left(1-\alpha_{i}\right) \omega_{i}+k_{i}^{*}(\alpha, \omega) \sum_{j \neq i}\left(1-\alpha_{j}\right) \omega_{j}\right\}}\right] \\
& =h\left(\alpha_{i}\right)=\alpha_{i} \ln \alpha_{i} /\left(\alpha_{i}-1\right) .
\end{aligned}
$$

Thus, $k_{i}^{*}(\alpha, \omega)=e^{h\left(\alpha_{i}\right)}\left(1-\alpha_{i}\right) \omega_{i} /\left\{\left(e^{h\left(\alpha_{i}\right)}-1\right) \sum_{j=1}^{n}\left(1-\alpha_{j}\right) \omega_{j}\right\}$. Notice that $\Delta u_{i}(\alpha, \omega, k)$ is strictly decreasing in $k$ :

$$
\frac{\partial}{\partial k} \Delta u_{i}(\alpha, \omega, k)=-\frac{\left(1-\alpha_{i}\right)^{2} \omega_{i}}{k\left[(k-1)\left(1-\alpha_{i}\right) \omega_{i}+k \sum_{j \neq i}\left(1-\alpha_{j}\right) \omega_{j}\right]}<0 .
$$

Therefore, $\Delta u_{i}(\alpha, \omega, k) \stackrel{\lesseqgtr}{>} 0$ if and only if $k \underset{<}{\gtrless} k_{i}^{*}(\alpha, \omega)$. This implies the desired result.

Theorem 3 implies that for any Cobb-Douglas economy $(\alpha, \omega) \in E^{C D}$, no agent has a participation incentive in a sufficiently large replica of the economy. Figure 3 illustrates the result in Theorem 3 when there are $n=2$ agents, each agent has the same endowment, $\omega_{1}=\omega_{2}$, and the number of replication is $k=1,2$, and 5. As $k$ increases, the region of preference parameters $\left(\alpha_{1}, \alpha_{2}\right)$ for which $\Delta u_{1}<0$ and $\Delta u_{2}<0$ becomes larger and it converges to the entire space $(0,1) \times(0,1)$. In other words, the measure of the set of Cobb-Dogulas utility economies for which at least one of two agents has a participation incentive vanishes as the replication size grows large. That is, the participation incentive disappears in a large economy. This could be interpreted as another support for Olson (1965) conjecture that a public good would be less likely provided as the number of agents increases.

A similar negative result holds for quasi-linear preferences. Given a quasi-linear utility economy $(\beta, \omega) \in E^{Q L}$ consisting of $n$-type agents, we consider the $k$-replica of this economy in which there are $k$ agents of type $\left(\beta_{i}, \omega_{i}\right)$ for each $i \in N$. For each agent of type $\left(\beta_{i}, \omega_{i}\right)$, let 


$$
\Delta u_{i}(\beta, \omega, k) \equiv u_{i}^{\beta_{i}}\left(x_{i}^{k N}, y^{k N}\right)-u_{i}^{\beta_{i}}\left(\omega_{i}, y^{k N-\{i\}}\right)
$$

where $\left(x_{i}^{k N}, y^{k N}\right)$ is the consumption bundle each agent of type $\left(\beta_{i}, \omega_{i}\right)$ receives at the unique Lindahl allocation when all agents participate in a Lindahl mechanism and $y^{k N-\{i\}}$ is the public good level produced at the unique Lindahl allocation when all agents except one of agents of type $\left(\beta_{i}, \omega_{i}\right)$ participate in the mechanism in the $k$-replica economy. We have the following result:

Theorem 4 Consider an arbitrary Lindahl mechanism $\Gamma$ under $\mu$. Given any $n \geq 2$, any $(\beta, \omega) \in E^{Q L}$, and any $i \in N, \Delta u_{i}(\beta, \omega, 1) \underset{<}{\gtrless}$ if and only if $\beta_{i} \underset{<}{\gtrless}(e-1)$ $\sum_{j \neq i} \beta_{j}$, where $e$ is the base of the natural logarithm; and $\Delta u_{i}(\beta, \omega, k)<0$ for any positive integer $k \geq 2$.

Proof It is not difficult to see that $\left(x_{i}^{k N}, y^{k N}\right)=\left(\omega_{i}-\beta_{i}, k \sum_{j \in N} \beta_{j}\right)$ and $y^{k N-\{i\}}=$ $(k-1) \beta_{i}+k \sum_{j \neq i} \beta_{j}$. Hence,

$$
\Delta u_{i}(\beta, \omega, k)=\beta_{i}\left\{-1+\ln \left[k \beta_{i}+k \sum_{j \neq i} \beta_{j}\right]-\ln \left[(k-1) \beta_{i}+k \sum_{j \neq i} \beta_{j}\right]\right\} .
$$

Let $k_{i}^{*}(\beta, \omega)$ be a value satisfying the equation $\Delta u_{i}\left(\beta, \omega, k_{i}^{*}(\beta, \omega)\right)=0$. This equation can be rewritten as $\ln \left[\left\{k_{i}^{*}(\beta, \omega) \beta_{i}+k_{i}^{*}(\beta, \omega) \sum_{j \neq i} \beta_{j}\right\} /\left\{\left(k_{i}^{*}(\beta, \omega)-1\right) \beta_{i}+\right.\right.$ $\left.\left.k_{i}^{*}(\beta, \omega) \sum_{j \neq i} \beta_{j}\right\}\right]=1$. Therefore, $k_{i}^{*}(\beta, \omega)=e \beta_{i} /\left\{(e-1) \sum_{j=1}^{n} \beta_{j}\right\}$. Also, note that $\Delta u_{i}(\beta, \omega, k)$ is strictly decreasing in $k$ :

$$
\frac{\partial}{\partial k} \Delta u_{i}(\beta, \omega, k)=-\frac{\beta_{i}^{2}}{k\left[(k-1) \beta_{i}+k \sum_{j \neq i} \beta_{j}\right]}<0
$$

Hence, $\Delta u_{i}(\beta, \omega, k) \gtreqless 0$ if and only if $k \leqq k_{i}^{*}(\beta, \omega)$. Let $k=1$. Then $\Delta u_{i}(\beta, \omega, 1) \stackrel{\gtrless}{<} 0$ if and only if $\beta_{i} \underset{<}{\rightleftharpoons}(e-1) \sum_{j \neq i} \beta_{j}$. On the other hand, if $k \geq 2$, then $k>k_{i}^{*}(\beta, \omega)=$ $e \beta_{i} /\left\{(e-1) \sum_{j=1}^{n} \beta_{j}\right\}$, so that $\Delta u_{i}(\beta, \omega, k)<0$.

Figure 4 illustrates the result in Theorem 4 for the case of two agents, $n=2$ and no replication, $k=1$. In this case, it follows from Theorem 4 that $\Delta u_{1}(\beta, \omega, 1) \underset{<}{\gtrless} 0$ if and only if $\beta_{2} \leqq \beta_{1} /(e-1)$ and $\Delta u_{2}(\beta, \omega, 1) \gtreqless 0$ if and only if $\beta_{2} \gtreqless(e-1) \beta_{1}$. Notice there is no possibility for which $\Delta u_{i}(\beta, \omega, 1)>0$ holds for all $i \in\{1,2\}$, that is, both agents have participation incentives. In other words, the measure of the set of economies for which the voluntary participation condition is satisfied is zero. No replication of an economy is necessary to obtain this negative result. Moreover, for the just $k=2$-replica of any economy $(\beta, \omega) \in E^{Q L}, \Delta u_{i}(\beta, \omega, 2)<0$ holds for any $i \in\{1,2\}$, that is, no agent has a participation incentive. This negative conclusion 
Fig. 4 Participation incentives in two-agent quasi-linear economies: the $k=1$-replica case

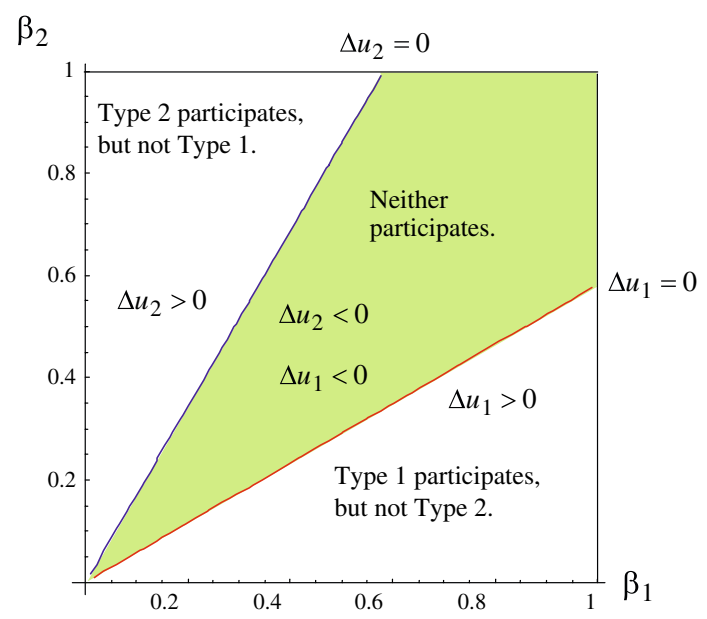

holds for at least two-replica of an arbitrary quasi-linear utility economy. In this sense, the result for quasi-linear utility economies is stronger than that for Cobb-Douglas utility economies.

\section{Impossibility results on voluntary participation in general mechanisms}

So far we have limited our attentions to Lindahl mechanisms, which satisfy individual rationality and Pareto efficiency, in Cobb-Douglas and quasi-linear utility economies. However, neither Pareto efficiency nor individual rationality is necessary to obtain a negative result on voluntary participation. In this section, we consider a large class of mechanisms that are necessarily neither individually rational nor Pareto efficient, including the voluntary contribution mechanism. We establish impossibility results that no mechanisms satisfy the voluntary participation condition on a sufficiently large domain of economies.

\subsection{The voluntary contribution mechanism}

First, let us study the voluntary contribution mechanism that does not satisfy Pareto efficiency when the equilibrium concept is Nash equilibrium. We will find that this mechanism fails to meet the voluntary participation condition, even though the name of the mechanism contains the term "voluntary".

Definition 2 The voluntary contribution mechanism is a mechanism such that for all $T \in P(N)$ and $i \in T, S_{i}^{T}=\left[0, \omega_{i}\right]$ and $g_{i}^{T}(s)=\left(\omega_{i}-s_{i}, \sum_{i \in T} s_{i}\right)$ for $s \in S^{T}$.

The above definition of the voluntary contribution mechanism is a generalization of the usual one, in which all agents are supposed to participate, to the case in which voluntary participation is allowed. When the equilibrium concept is Nash equilibrium, 
Fig. 5 The proof of Lemma 1

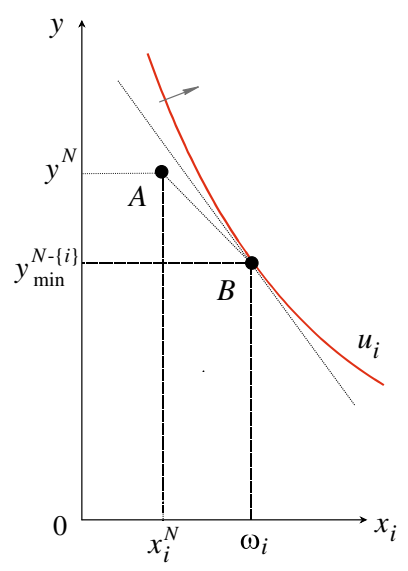

each agent $i$ selects her contribution out of her endowment to the provision of the public good, $s_{i}$, to maximize her utility $u_{i}\left(\omega_{i}-s_{i}, \sum_{j \in T} s_{j}\right)$, given contributions of the other agents in $T,\left(s_{j}\right)_{j \in T-\{i\}}$ in the voluntary contribution mechanism.

We begin by proving the following result that is simple, but useful below. Let an equilibrium correspondence $\mu$ be given.

Lemma 1 Suppose that a mechanism $\Gamma$ and an economy $(u, \omega)$ satisfy the following condition: for some $\left(x^{N}, y^{N}\right) \in g^{N} \circ \mu_{\Gamma}\left(e_{N}\right)$ and some $i \in N$,

$$
\frac{y^{N}-y_{\min }^{N-\{i\}}}{\omega_{i}-x_{i}^{N}} \leq \operatorname{MRS}_{i}\left(\omega_{i}, y_{\min }^{N-\{i\}}\right)
$$

where $\operatorname{MRS}_{i}\left(\omega_{i}, y_{\min }^{N-\{i\}}\right) \equiv \frac{\partial u_{i}\left(\omega_{i}, y_{\min }^{N-\{i\}}\right)}{\partial x_{i}} / \frac{\partial u_{i}\left(\omega_{i}, y_{\min }^{N-\{i\}}\right)}{\partial y}$ is agent $i$ 's marginal rate of substitution at $\left(\omega_{i}, y_{\min }^{N-\{i\}}\right)$. Then the mechanism $\Gamma$ fails to satisfy voluntary participation for the economy $(u, \omega)$ under $\mu$.

Proof The basic idea behind the proof is illustrated in Fig. 5 in which the horizontal axis denotes agent $i$ 's consumption level of private good $x_{i}$, and the vertical axis stands for the public good level, $y$. The above inequality (6.1) says that the slope of the line going through Point $A=\left(\omega_{i}, y_{\min }^{N-\{i\}}\right)$ and Point $B=\left(\omega_{i}, y_{\min }^{N-\{i\}}\right)$ is smaller than or equal to the slope of the tangent to agent $i$ 's indifference curve, that is, the marginal rate of substitution at $B=\left(\omega_{i}, y_{\min }^{N-\{i\}}\right)$. By strict quasi-concavity of $u_{i}$, agent $i$ strictly prefers Point B to Point A, so that she fails to have the participation incentive in this economy.

The usefulness of Lemma 1 is that it is applicable to any mechanism and any utility function form. Firs of all, we apply Lemma 1 to the voluntary contribution mechanism and any Cobb-Douglas utility economy. In what follows, we assume that for all $i \in N$, 


$$
\omega_{i}>\frac{\alpha_{i} \sum_{j \neq i} \omega_{j}}{\left(1-\alpha_{i}\right)\left\{1+\sum_{j \neq i}\left[\alpha_{j} /\left(1-\alpha_{j}\right)\right]\right\}}
$$

to ensure an interior equilibrium. ${ }^{8}$ We have the following negative result:

Theorem 5 Let $(\alpha, \omega) \in E^{C D}$ be any Cobb-Douglas utility economy in which $\alpha_{i} \geq$ $1 / n$ for agent $i$ such that $\omega_{i} \leq \omega_{j}$ for all $j \neq i$.Then the voluntary contribution mechanism fails to satisfy voluntary participation for $(\alpha, \omega)$ under the Nash equilibrium correspondence.

The proof of Theorem 5 is in the appendix. Theorem 5 says that if the agent whose endowment is the smallest has a sufficiently large value of the private good relative to the public good (i.e., $\alpha_{i} \geq 1 / n$ ), then she loses her participation incentive in the voluntary contribution mechanism. Notice that the region of $\alpha_{i}$ for which the voluntary participation condition is violated (that is, $\alpha_{i} \geq 1 / n$ ) expands as the number of agents $n$ grows larger. This result is interpretable as another support of Olson's conjecture that a public good would be less likely provided as the size of an economy becomes larger.

A similar negative result holds for any quasi-linear utility economy. Given a quasilinear utility economy $(\beta, \omega) \in E^{Q L}$ and $T \in P(N)$, let $\beta_{\max }^{T} \equiv \max _{i \in T} \beta_{i}$ be the maximal value of $\beta_{i}$ among the agents in $T$ and $M^{T} \equiv\left\{i \in T \mid \beta_{i}=\beta_{\max }^{T}\right\}$ be the set of agents who have the maximal value $\beta_{\max }^{T}$. If $\# M^{T} \geq 2$, then there are multiple Nash equilibrium allocations in which the public good level and the sum of contributions by the agents belonging to $M^{T}$ are equal to $\beta_{\max }^{T}$.

Theorem 6 Let $(\beta, \omega) \in E^{Q L}$ be any quasi-linear utility economy in which either $(i)$ $\# M^{N} \geq 2$, or (ii) $M^{N}=\{i\}$ and $\beta_{i}<e \cdot \beta_{\max }^{N-\{i\}}$, where $e$ is the base of the natural logarithm. Then the voluntary contribution mechanism fails to satisfy the voluntary participation condition for $(\beta, \omega)$ under the Nash equilibrium correspondence.

The proof of Theorem 6 is in the appendix. Theorem 6 means the following. First, when $\# M^{N} \geq 2$, at least one agent in $\# M^{N}$, say agent 1 , makes a positive contribution at each of Nash equilibria. If agent 1 chooses non-participation, then her contribution becomes zero, while the public good level is unchanged and provided by the other agents in $M^{N}$, so that agent 1 becomes better off. ${ }^{9}$ Second, when $M^{N}=\{i\}$, there is a unique equilibrium allocation in which the public good level is $\beta_{i}$ and only agent $i$ contributes $\beta_{i}$ to the public good. By deciding not to participate in the mechanism, agent $i$ does not have to make any contribution, whereas the public good level becomes

\footnotetext{
${ }^{8}$ It is easy to check that each agent $i$ 's contribution to the public good in the voluntary contribution mechanism for a Cobb-Doulas utility economy $(\alpha, \omega) \in E^{C D}$ when all agents participate is provided by $\omega_{i}-x_{i}^{N}=\frac{1+\sum_{j \neq i}\left[\alpha_{j} /\left(1-\alpha_{j}\right)\right]}{1+\sum_{\ell=1}^{n}\left[\alpha_{\ell} /\left(1-\alpha_{\ell}\right)\right]}\left(\omega_{i}-\frac{\alpha_{i} \sum_{j \neq i} \omega_{j}}{\left(1-\alpha_{i}\right)\left\{1+\sum_{j \neq i}\left[\alpha_{j} /\left(1-\alpha_{j}\right)\right]\right\}}\right), \quad i \in N$, which is positive by (6.2), increasing in $\omega_{i}$, and decreasing in $\alpha_{i}$.

9 Notice that the mechanism does not satisfy the voluntary participation condition if in at least one of equilibrium allocations, some agent loses a participation incentive. However, we have a stronger result: at each of the equilibrium allocations, there is some agent who will not participate in the mechanism. See the proof of Theorem 6 .
} 
$\beta_{\max }^{N-\{i\}}$. If this new public good level is sufficiently large (i.e., $\beta_{i}<e \cdot \beta_{\max }^{N-\{i\}}$ ), then agent $i$ has a non-participation incentive.

By Theorem 5 (resp. 6), if the class of admissible economies $E$ contains a CobbDouglas (resp. quasi-linear) utility economy satisfying the condition in the theorem, then the voluntary contribution mechanism fails to satisfy the voluntary participation condition on $E$.

\subsection{General mechanisms}

In this section we will consider a large class of mechanisms and show impossibility results on voluntary participation in mechanisms in the class. First, we investigate the case of two agents. We impose the following mild conditions on a mechanism.

Definition 3 The mechanism $\Gamma$ satisfies non-emptiness for an economy e under $\mu$ if for all $T \in P(N), g^{T} \circ \mu_{\Gamma}\left(e_{T}\right) \neq \varnothing$.

Definition 4 The mechanism $\Gamma$ satisfies feasibility for an economy e under $\mu$ if for all $T \in P(N), g^{T} \circ \mu_{\Gamma}\left(e_{T}\right) \subseteq A^{T}$.

Non-emptiness says that there should always exist an equilibrium. Feasibility demands that every equilibrium allocation of the mechanism be feasible. Note that we require feasibility only at equilibrium, but not out of equilibrium. Moreover, a feasible mechanism does not necessarily satisfy individual feasibility (i.e., for all $T \in P(N)$ and all $s \in S^{T}, g^{T}(s) \in \Re_{+}^{\# T+1}$ ) nor balancedness (i.e., for all $T \in P(N)$ and all $\left.s \in S^{T}, g^{T}(s) \in A^{T}\right)$.

Definition 5 The mechanism $\Gamma$ satisfies the Robinson Crusoe condition for an economy $e=(u, w)$ under $\mu$ if for all $i \in N$, if $\left(x_{i}^{\{i\}}, y^{\{i\}}\right) \in g^{\{i\}} \circ \mu_{\Gamma}\left(e_{i}\right)$, then $\left(x_{i}^{\{i\}}, y^{\{i\}}\right) \in \underset{\left(x_{i}, y\right) \in A^{\{i\}}}{\operatorname{Arg} \max } u_{i}\left(x_{i}, y\right)$.

The Robinson Crusoe condition means that if only one agent participates in the mechanism, then she chooses an outcome that is best for her. Clearly, any Lindahl mechanism and the voluntary contribution mechanism satisfy this condition.

We have the following two-agent impossibility theorems on voluntary participation:

Theorem 7 Let $n=2$ and $(\alpha, \omega) \in E^{C D}$ be any Cobb-Douglas utility economy in which $\alpha_{i}>0.5, i=1,2$, and $\left(1-\alpha_{1}\right) \omega_{1}=\left(1-\alpha_{2}\right) \omega_{2}$. If a mechanism satisfies nonemptiness, feasibility, and the Robinson Crusoe condition for $(\alpha, \omega)$ under $\mu$, then it fails to satisfy the voluntary participation condition for $(\alpha, \omega)$ under $\mu$.

Theorem 8 Let $n=2$ and $(\beta, \omega) \in E^{Q L}$ be any quasi-linear utility economy in which $\beta_{i}<e^{1 / 2} \beta_{j}$ for agent $i$ such that $\beta_{i}>\beta_{j}, j \neq i$, where $e$ is the base of the natural logarithm. If a mechanism satisfies non-emptiness, feasibility, and the Robinson Crusoe condition for $(\beta, \omega)$ under $\mu$, then it fails to satisfy the voluntary participation condition for $(\beta, \omega)$ under $\mu$. 
The proofs of Theorems 7 and 8 are in the appendix. Both Theorems 7 and 8 can be applied to any mechanism, whereas the condition in Theorem 7 (resp. 8) for which the voluntary participation condition is violated is stronger than that in Theorem 1 (resp. 2) focusing on any Lindahl mechanism as well as that in Theorem 5 (resp. 6) for the voluntary contribution mechanism in the case of two agents with Cobb-Douglas (resp. quasi-linear) utility economies.

Finally, we examine the general case of at least two agents. We introduce the following condition on a mechanism:

Definition 6 The mechanism $\Gamma$ satisfies contribution monotonicity for an economy $e=(u, \omega)$ under $\mu$ if for all $\left(x^{N}, y^{N}\right) \in g^{N} \circ \mu_{\Gamma}\left(e_{N}\right)$, all $i \in N$, and all $\left(x^{N-\{i\}}\right.$, $\left.y^{N-\{i\}}\right) \in g^{N-\{i\}} \circ \mu_{\Gamma}\left(e_{N-\{i\}}\right), \sum_{j \neq i} \omega_{j}-x_{j}^{N} \leq \sum_{j \neq i} \omega_{j}-x_{j}^{N-\{i\}}$.

Contribution monotonicity means the following. Suppose that $n-1$ agents except $i$ initially participate in the mechanism, and each of those $n-1$ participants contributes $\omega_{j}-x_{j}^{N-\{i\}}$ of the private good to provide the public good at equilibrium. Now imagine that the non-participant $i$ also participates, so that the equilibrium contribution by each of the previous $n-1$ participants becomes $\omega_{j}-x_{j}^{N}$. Then contribution monotonicity requires that the sum of equilibrium contributions by the $n-1$ participants should not increase, that is, $\sum_{j \neq i} \omega_{j}-x_{j}^{N} \leq \sum_{j \neq i} \omega_{j}-x_{j}^{N-\{i\}}$. Roughly speaking, contribution monotonicity means that the burdens by participants in the mechanism do not rise as the number of agents who choose participation become larger. It is not hard to check that any Lindahl mechanism and the voluntary participation mechanism satisfy contribution monotonicity for any Cobb-Douglas or quasi-linear utility economy.

By applying Lemma 1, we have the following impossibility result:

Theorem 9 Suppose that (i) a mechanism $\Gamma$ satisfies non-emptiness, feasibility, and contribution monotonicity for an economy $(u, \omega)$ under $\mu$; and (ii) $1 \leq \frac{\partial u_{i}\left(\omega_{i}, y_{\min }^{N-\{i\}}\right)}{\partial x_{i}} /$ $\frac{\partial u_{i}\left(\omega_{i}, y_{\min }^{N-\{i\}}\right)}{\partial y}$ for some $i$. Then $\Gamma$ fails to satisfy voluntary participation for $(u, \omega)$ under $\mu$.

The proof of Theorem 9 is in the appendix. By Theorem 9, there is no mechanism satisfying non-emptiness, feasibility, contribution monotonicity, and voluntary participation on a domain of economies $E$ if $E$ contains an economy $(u, \omega)$ satisfying the condition in Theorem 9. Theorem 9 is applicable to any mechanism and any utility function, but the condition for which the voluntary condition is not satisfied is stronger than that in Theorem 1 for Lindahl mechanisms and Cobb-Douglas utility economies. ${ }^{10}$ This is because Theorem 1 is derived by comparing the utility level of participation with that of non-participation directly, whereas Theorem 9 is obtained by

10 For any Lindahl mechanism and any Cobb-Dogulass economy, it is straightforward to check that the condition (ii) in Theorem 9 holds if $\alpha_{i} \geq 1 /(n-1)$ for agent $i$ such that $\left(1-\alpha_{i}\right) \omega_{i} \leq\left(1-\alpha_{j}\right) \omega_{j}$ for all $j \neq i$, which is stronger than the condition $\alpha_{i}>\alpha(n)$ in Theorem 1. 
using only local information on a utility function, i.e., the marginal rate substitution (see Fig. 5 in Lemma 1). ${ }^{11}$

\section{Concluding remarks}

We see that the solutions to the free-rider problem, which have been proposed in mechanism design theory, are not necessary solutions to the free-rider problem when participation in mechanisms is voluntary. It is quite difficult or impossible to design mechanisms with voluntary participation: any reasonable mechanism, including any Lindhal mechanism and the voluntary contribution mechanism, fails to satisfy the voluntary participation condition on a sufficiently large domain of economies including Cobb-Douglas or quasi-linear economies.

There are several open questions to examine. First, we assumed that preferences and endowments are mutually known among agents, although the equilibrium notion is arbitrary as long as it is consistent with the complete information assumption. ${ }^{12}$ It remains to investigate the voluntary participation problem when the agents do not know the preferences of the others by using an equilibrium concept with incomplete information such as Bayesian Nash equilibrium.

Second, we assumed that all agents can access the technology to the production of the public good freely. Instead, if only the mechanism designer is able to use the production technology and cancel a mechanism (that is, produce no public good) unless all agents participate, then a mechanism satisfying the standard individual rationality condition should work well. However, this is possible only if the designer have enough power to force all agents not to produce the public good. It is an open question to examine this issue.

Finally, Cason et al. (2002) and Cason et al. (2004) observed that cooperation has emerged though spiteful behavior in their experiments on the voluntary contribution mechanism with voluntary participation. Our theory in this paper suggests that no cooperation will emerge. Reconciling theoretical results to experimental results is an open area of our future research.

Open Access This article is distributed under the terms of the Creative Commons Attribution Noncommercial License which permits any noncommercial use, distribution, and reproduction in any medium, provided the original author(s) and source are credited.

\footnotetext{
11 On the other hand, for any Lindahl mechanism and any quasi-linear utility economy, it is easy to see that the condition (ii) in Theorem 9 always holds regardless of the values of preference parameters, because $\operatorname{MRS}_{i}\left(\omega_{i}, y_{\min }^{N-\{i\}}\right)=\sum_{j \neq i} \beta_{j} / \beta_{i} \geq n-1 \geq 1$ for agent $i$ such that $\beta_{i} \leq \beta_{j}$ for all $j \neq i$. The result is the same as that in Theorem 2, and Theorem 2 can be obtained as a corollary of Theorem 9, although the proofs are quite different.

12 Nevertheless, even when the utility functions are privately known, a Nash equilibrium can be interpreted as a rest point of the dynamic learning process (Hurwicz 1972). In fact, Nash equilibrium outcomes have been observed in economic experiments even in incomplete information environments (Cason et al. 2006).
} 


\section{Appendix}

Proof of Theorem 5 Fix any $(\alpha, \omega) \in E^{C D}$. For each $T \in P(N)$, it is easy to check that there is a unique Nash equilibrium allocation of the voluntary contribution mechanism for $\left(\alpha_{i}, \omega_{i}\right)_{i \in T}$ when agents in $T$ participate in it, given by

$$
\left(x_{i}^{T}, y^{T}\right)=\left(\frac{\alpha_{i} \sum_{\ell \in T} \omega_{\ell}}{\left(1-\alpha_{i}\right)\left\{1+\sum_{\ell \in T}\left[\alpha_{\ell} /\left(1-\alpha_{\ell}\right)\right]\right\}}, \frac{\sum_{\ell \in T} \omega_{\ell}}{\left\{1+\sum_{\ell \in T}\left[\alpha_{\ell} /\left(1-\alpha_{\ell}\right)\right]\right\}}\right)
$$

for $i \in T$. Pick agent $i$ such that $\omega_{i} \leq \omega_{j}$ for all $j \neq i$. Since $(n-1) \omega_{i} \leq \sum_{j \neq i} \omega_{j}$,

$$
\begin{aligned}
\operatorname{MRS}_{i}\left(\omega_{i}, y_{\min }^{N-\{i\}}\right) & =\frac{\alpha_{i} \sum_{j \neq i} \omega_{j}}{\omega_{i}\left(1-\alpha_{i}\right)\left\{1+\sum_{j \neq i}\left[\alpha_{j} /\left(1-\alpha_{j}\right)\right]\right\}} \\
& \geq \frac{\alpha_{i}(n-1)}{\left(1-\alpha_{i}\right)\left\{1+\sum_{j \neq i}\left[\alpha_{j} /\left(1-\alpha_{j}\right)\right]\right\}}
\end{aligned}
$$

Also, $\frac{y^{N}-y_{\min }^{N-\{i\}}}{\omega_{i}-x_{i}^{N}}=\frac{1}{\left\{1+\sum_{j \neq i}\left[\alpha_{j} /\left(1-\alpha_{j}\right)\right]\right\}}$. Therefore, $\frac{y^{N}-y_{\min }^{N-\{i\}}}{\omega_{i}-x_{i}^{N}} \leq \operatorname{MRS} S_{i}\left(\omega_{i}, y_{\min }^{N-\{i\}}\right)$ if $\alpha_{i} \geq 1 / n$. By Lemma 1 , we have the desired result.

Proof of Theorem 6 Fix any $(\beta, \omega) \in E^{Q L}$. For each $T \in P(N)$, it is easy to check that the set of Nash equilibrium allocations of the voluntary contribution mechanism for $\left(\beta_{i}, \omega_{i}\right)_{i \in T}$ when agents in $T$ participate in it, $g^{T} \circ \mu_{\Gamma}\left(e_{T}\right)$, is given by

$$
\begin{aligned}
& \left\{\left(\left(x_{i}\right)_{i \in T}, y\right) \in \mathfrak{R}_{+}^{\# T+1} \mid y=\sum_{i \in T}\left(\omega_{i}-x_{i}\right)=\beta_{\max }^{T}, \quad x_{i} \in\left[\omega_{i}-\beta_{\max }^{T}, \omega_{i}\right],\right. \\
& \left.\forall i \in M^{T}, x_{j}=\omega_{j}, \forall j \notin M^{T}\right\} .
\end{aligned}
$$

There are multiple Nash equilibria unless $\# M^{T}=1$, but the equilibrium level of the public good is always uniquely determined.

Let $\left(\left(x_{i}^{N}\right)_{i \in N}, y^{N}\right) \in g^{N} \circ \mu_{\Gamma}\left(e_{N}\right)$ be given. First, suppose that $\# M^{N} \geq 2$. Notice that there is some agent $i \in M^{N}$ such that $x_{i}^{N}<\omega_{i}$ and $y^{N}=y^{N-\{i\}}=\beta_{\max }^{N}$. Since $\operatorname{MRS}_{i}\left(\omega_{i}, y_{\min }^{N-\{i\}}\right)=\beta_{\max }^{N} / \beta_{i}=1>0=\frac{y^{N}-y_{\min }^{N-\{i\}}}{\omega_{i}-x_{i}^{N}}$, it follows from Lemma 1 that the voluntary participation condition is not satisfied.

Second, suppose that $M^{N}=\{i\}$ and $\beta_{i}<e \cdot \beta_{\max }^{N-\{i\}}$. Then $x_{i}^{N}=\omega_{i}-\beta_{\max }^{N}=$ $\omega_{i}-\beta_{i}, y^{N}=\beta_{\max }^{N}=\beta_{i}$, and $y^{N-\{i\}}=\beta_{\max }^{N-\{i\}}$. Since $\beta_{i}<e \cdot \beta_{\max }^{N-\{i\}}, \Delta u_{i}(\beta)=$ $u_{i}^{\beta_{i}}\left(x_{i}^{N}, y^{N}\right)-u_{i}^{\beta_{i}}\left(\omega_{i}, y^{N-\{i\}}\right)=-\beta_{i}\left\{1-\ln \left[\beta_{i} / \beta_{\max }^{N-\{i\}}\right]\right\}<0$. Thus we have the desired result. 
Proof of Theorem 7 Suppose by way of contradiction that the mechanism satisfies the voluntary participation condition. It is easy to check that by the Robinson Crusoe condition, a unique equilibrium allocation of the mechanism for one agent CobbDouglas utility economy is given by $\left(x_{i}^{\{i\}}, y^{\{i\}}\right)=\left(\alpha_{i} \omega_{i},\left(1-\alpha_{i}\right) \omega_{i}\right), i=1,2$. Let $V\left(\left(\omega_{i}, y^{\{j\}}\right), u_{i}^{\alpha_{i}}\right) \equiv\left\{\left(x_{i}, y\right) \in \Re_{+}^{2} \mid u_{i}^{\alpha_{i}}\left(x_{i}, y\right) \geq u_{i}^{\alpha_{i}}\left(\omega_{i}, y^{\{j\}}\right)\right\}$ be agent $i$ 's weak upper contour set at $\left(\omega_{i}, y^{\{j\}}\right)$ for $u_{i}^{\alpha_{i}}$, where $\left(\omega_{i}, y^{\{j\}}\right)=\left(\omega_{i},\left(1-\alpha_{j}\right) \omega_{j}\right)$ and $j \neq i$. Pick any $\left(x_{1}^{\{1,2\}}, x_{2}^{\{1,2\}}, y^{\{1,2\}}\right) \in g^{\{1,2\}} \circ \mu_{\Gamma}\left(u_{1}^{\alpha_{1}}, u_{2}^{\alpha_{2}}\right)$. By the voluntary participation condition,

$$
\left(x_{i}^{\{1,2\}}, y^{\{1,2\}}\right) \in V\left(\left(\omega_{i}, y^{\{j\}}\right), u_{i}^{\alpha_{i}}\right) \quad(i, j=1,2 ; j \neq i) .
$$

We claim that

$$
\forall\left(x_{i}, y\right) \in V\left(\left(\omega_{i}, y^{\{j\}}\right), u_{i}^{\alpha_{i}}\right), 2 x_{i}+y>2 \omega_{i} \quad(i, j=1,2 ; j \neq i) .
$$

Suppose that (6.4) does not hold. Then for some $i$ and some $\left(\bar{x}_{i}, \bar{y}\right) \in \mathfrak{R}_{+}^{2}, u_{i}^{\alpha_{i}}\left(\bar{x}_{i}, \bar{y}\right) \geq$ $u_{i}^{\alpha_{i}}\left(\omega_{i}, y^{\{j\}}\right)$ and $2 \bar{x}_{i}+\bar{y} \leq 2 \omega_{i}$. Let $\left(x_{i}^{*}, y^{*}\right)$ be a maximizer of the utility function $u_{i}^{\alpha_{i}}\left(x_{i}, y\right)=\alpha_{i} \ln x_{i}+\left(1-\alpha_{i}\right) \ln y$ subject to the constraint $2 x_{i}+y \leq 2 \omega_{i}$. It is easy to see that $\left(x_{i}^{*}, y^{*}\right)=\left(\alpha_{i} \omega_{i}, 2\left(1-\alpha_{i}\right) \omega_{i}\right)$ and $u_{i}^{\alpha_{i}}\left(x_{i}^{*}, y^{*}\right)-u_{i}^{\alpha_{i}}\left(\omega_{i}, y^{\{j\}}\right)=\alpha_{i} \ln \alpha_{i}+$ $\left(1-\alpha_{i}\right) \ln \left(2\left(1-\alpha_{i}\right) \omega_{i} /\left(1-\alpha_{j}\right) \omega_{j}\right)=\alpha_{i} \ln \alpha_{i}+\left(1-\alpha_{i}\right) \ln 2<0$ since $\left(1-\alpha_{i}\right) \omega_{i}=$ $\left(1-\alpha_{j}\right) \omega_{j}$ and $\alpha_{i}>0.5$. Thus, $u_{i}^{\alpha_{i}}\left(\bar{x}_{i}, \bar{y}\right) \geq u_{i}^{\alpha_{i}}\left(\omega_{i}, y^{\{j\}}\right)>u_{i}^{\alpha_{i}}\left(x_{i}^{*}, y^{*}\right)$, which contradicts the fact that $\left(x_{i}^{*}, y^{*}\right)$ is the maximizer of $u_{i}^{\alpha_{i}}\left(x_{i}, y\right)$ subject to $2 x_{i}+y \leq 2 \omega_{i}$.

However, by (6.3) and (6.4), $x_{1}^{\{1,2\}}+x_{2}^{\{1,2\}}+y^{\{1,2\}}>\omega_{1}+\omega_{2}$. This contradicts the feasibility condition on the mechanism.

Proof of Theorem 8 Suppose by way of contradiction that the mechanism satisfies the voluntary participation condition. It is easy to see that by the Robinson Crusoe condition, a unique equilibrium allocation of the mechanism for one agent quasilinear utility economy is given by $\left(x_{i}^{\{i\}}, y^{\{i\}}\right)=\left(\omega_{i}-\beta_{i}, \beta_{i}\right), i=1,2$. Let $V\left(\left(\omega_{i}, y^{\{j\}}\right), u_{i}^{\beta_{i}}\right) \equiv\left\{\left(x_{i}, y\right) \in \Re_{+}^{2} \mid u_{i}^{\beta_{i}}\left(x_{i}, y\right) \geq u_{i}^{\beta_{i}}\left(\omega_{i}, y^{\{j\}}\right)\right\}$ be agent $i$ 's weak upper contour set at $\left(\omega_{i}, y^{\{j\}}\right)$ for $u_{i}^{\beta_{i}}$, where $\left(\omega_{i}, y^{\{j\}}\right)=\left(\omega_{i}, \beta_{j}\right)$ and $j \neq i$. Pick any $\left(x_{1}^{\{1,2\}}, x_{2}^{\{1,2\}}, y^{\{1,2\}}\right) \in g^{\{1,2\}} \circ \mu_{\Gamma}\left(u_{1}^{\beta_{1}}, u_{2}^{\beta_{2}}\right)$. By the voluntary participation condition,

$$
\left(x_{i}^{\{1,2\}}, y^{\{1,2\}}\right) \in V\left(\left(\omega_{i}, y^{\{j\}}\right), u_{i}^{\beta_{i}}\right) \quad(i, j=1,2 ; j \neq i) .
$$

We claim that

$$
\forall\left(x_{i}, y\right) \in V\left(\left(\omega_{i}, y^{\{j\}}\right), u_{i}^{\beta_{i}}\right), 2 x_{i}+y>2 \omega_{i} \quad(i, j=1,2 ; j \neq i) .
$$

Suppose that (6.6) does not hold. Then for some and some $\left(\bar{x}_{i}, \bar{y}\right) \in \Re_{+}^{2}, u_{i}^{\beta_{i}}\left(\bar{x}_{i}, \bar{y}\right) \geq$ $u_{i}^{\beta_{i}}\left(\omega_{i}, y^{\{j\}}\right)$ and $2 \bar{x}_{i}+\bar{y} \leq 2 \omega_{i}$. Let $\left(x_{i}^{*}, y^{*}\right)$ be a maximizer of the utility function 
$u_{i}^{\beta_{i}}\left(x_{i}, y\right)=x_{i}+\beta_{i} \ln y$ subject to the constraint $2 x_{i}+y \leq 2 \omega_{i}$. It is easy to check that $\left(x_{i}^{*}, y^{*}\right)=\left(\omega_{i}-\beta_{i} / 2, \beta_{i}\right)$ and $u_{i}^{\beta_{i}}\left(x_{i}^{*}, y^{*}\right)-u_{i}^{\beta_{i}}\left(\omega_{i}, y^{\{j\}}\right)=\beta_{i}\left[-1 / 2+\ln \left(\beta_{i} / \beta_{j}\right)\right]<0$, since $\beta_{i}<e^{1 / 2} \beta_{j}$ if $\beta_{i}>\beta_{j}$. Thus, $u_{i}^{\beta_{i}}\left(\bar{x}_{i}, \bar{y}\right) \geq u_{i}^{\beta_{i}}\left(\omega_{i}, y^{\{j\}}\right)>u_{i}^{\beta_{i}}\left(x_{i}^{*}, y^{*}\right)$, which is a contradiction.

However, by (6.5) and (6.6), $x_{1}^{\{1,2\}}+x_{2}^{\{1,2\}}+y^{\{1,2\}}>\omega_{1}+\omega_{2}$. This contradicts the feasibility condition on the mechanism.

Proof of Theorem 9 By feasibility and contribution monotonicity, $y^{N}-\left(\omega_{i}-x_{i}^{N}\right)=$ $\sum_{j \neq i}\left(\omega_{j}-x_{j}^{N}\right) \leq \sum_{j \neq i}\left(\omega_{j}-x_{j}^{N-\{i\}}\right)=y^{N-\{i\}}$ for any $\left(x^{N}, y^{N}\right) \in g^{N} \circ \mu_{\Gamma}\left(e_{N}\right)$ and any $\left(x^{N-\{i\}}, y^{N-\{i\}}\right) \in g^{N-\{i\}} \circ \mu_{\Gamma}\left(e_{N-\{i\}}\right)$. Therefore, $\frac{y^{N}-y_{\min }^{N-\{i\}}}{\omega_{i}-x_{i}^{N}} \leq 1$. Since $1 \leq \frac{\partial u_{i}\left(\omega_{i}, y_{\min }^{N-\{i\}}\right)}{\partial x_{i}} / \frac{\partial u_{i}\left(\omega_{i}, y_{\min }^{N-\{i\}}\right)}{\partial y}$, it follows from Lemma 1 that the voluntary participation condition is violated for $(u, \omega)$.

\section{References}

Cason T, Saijo T, Yamato T (2002) Voluntary participation and spite in public good provision experiments: an international comparison. Exp Econ 5:133-153

Cason TN, Saijo T, Yamato T, Yokotani K (2004) Non-excludable public good experiments. Games Econ Behav 49:81-102

Cason TN, Saijo T, Sjöström T, Yamato T (2006) Secure implementation experiments: do strategy-proof mechanisms really work? Games Econ Behav 57:206-235

Dixit A, Olson M (2000) Does voluntary participation undermine the Coase theorem? J Pub Econ 76: 309-335

Groves T, Ledyard J (1977) Optimal allocation of public goods: a solution to the 'Free Rider' problem. Econometrica 45:783-809

Hurwicz L (1972) On Informationally Decentralized Systems. In: Radner R, McGuire CB (eds) Decision and organization: a volume in honor of Jacob Marschak. North-Holland, Amsterdam pp 297-336

Hurwicz L (1979a) Outcome functions yielding Walrasian and Lindahl allocations at Nash equilibrium points. Rev Econ Stud 46:217-224

Hurwicz L (1979b) On allocations attainable through Nash equilibria. J Econ Theory 21:140-165

Hurwicz L, Maskin E, Postlewaite A (1984) Feasible Implementation of Social Choice Correspondences by Nash Equilibria, mimeo

Ledyard J, Roberts J (1974) On the incentive problem with public goods. Northwestern University, mimeo

Moore J, Repullo R (1988) Subgame perfect implementation. Econometrica 56:1191-1220

Moulin H (1986) Characterizations of the pivotal mechanism. J Pub Econ 31:53-78

Olson M (1965) The logic of collective action: public goods and the theory of groups. Harvard University Press, Cambridge

Palfrey T, Rosenthal H (1984) Participation and the provision of discrete public goods: a strategic analysis. J Pub Econ 24:171-193

Saijo T (1991) Incentive compatibility and individual rationality in public good economies. J Econ Theory 55:203-212

Saijo T, Yamato T (1999) A voluntary participation game with a non-excludable public good. J Econ Theory $84: 227-242$

Tian G (1990) Completely feasible and continuous implementation of the Lindahl correspondence with a message space of minimal dimension. J Econ Theory 51:443-452

Varian HR (1994) A Solution to the problem of externalities when agents are well-informed. Am Econ Rev 84:1278-1293

Walker M (1981) A simple incentive compatible scheme for attaining Lindahl allocations. Econometrica 49:65-71 